\title{
Intimate Partner Violence Screening Programs in the Veterans Health Administration: Informing Scale-up of Successful Practices
}

\author{
Katherine M. Iverson, $P h D^{1,2,3}$, Omonyêlé Adjognon, $S c M^{1,3}$, Alessandra R. Grillo, BS ${ }^{1}$, \\ Melissa E. Dichter, PhD, MSW ${ }^{4,5}$, Cassidy A. Gutner, $P h D^{1,2}$, \\ Alison B. Hamilton, PhD, MPH ${ }^{6,7}$, Shannon Wiltsey Stirman, PhD ${ }^{8,9}$, and Megan R. Gerber, \\ $\mathrm{MD}, \mathrm{MPH}^{10,11}$
}

\begin{abstract}
'Women's Health Sciences Division, National Center for PTSD, VA Boston Healthcare System, Boston, MA, USA; ${ }^{2}$ Department of Psychiatry, Boston University School of Medicine, Boston, MA, USA; ${ }^{3}$ Center for Healthcare Organization and Implementation Research (CHOIR), VA Boston Healthcare System, Boston, MA, USA; ${ }^{4}$ VA Center for Health Equity Research and Promotion (CHERP), Crescenz VA Medical Center, Philadelphia, PA, USA; ${ }^{5}$ School of Social Work, Temple University, Philadelphia, PA, USA; ${ }^{6}$ VA Center for the Study of Healthcare Innovation, Implementation and Policy (CSHIIP), VA Greater Los Angeles Healthcare System, Los Angeles, CA, USA; ' Department of Psychiatry and Biobehavioral Sciences, University of California Los Angeles David Geffen School of Medicine, Los Angeles, CA, USA; ${ }^{8}$ Dissemination and Training Division, National Center for PTSD, VA Palo Alto Healthcare System, Palo Alto, CA, USA; ${ }^{9}$ Department of Psychiatry and Behavioral Sciences, Stanford University School of

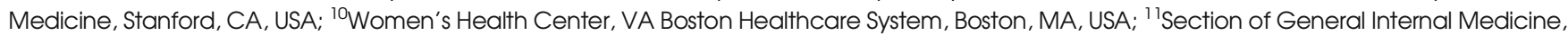
Boston University School of Medicine, Boston, MA, USA.
\end{abstract}

OBJECTIVES: Screening women for intimate partner violence (IPV) is increasingly expected in primary care, consistent with clinical prevention guidelines (e.g., United States Preventive Services Task Force). Yet, little is known about real-world implementation of clinical practices or contextual factors impacting IPV screening program success. This study identified successful clinical practices, and barriers to and facilitators of IPV screening program implementation in the Veterans Health Administration (VHA).

DESIGN: Descriptive, qualitative study of a purposeful sample of 11 Veterans Affairs Medical Centers (VAMCs) categorized as early and late adopters of IPV screening programs within women's health primary care clinics. VAMCs were categorized based on performance measures collected by VHA operations partners.

PARTICIPANTS: Thirty-two administrators and clinician key informants (e.g., Women's Health Medical Directors, IPV Coordinators, and physicians) involved in IPV screening program implementation decisions from six early- and five late-adopting sites nationwide.

MAIN MEASURES: Participants reported on IPV screening and response practices, and contextual factors impacting implementation, in individual 1 -h semi-structured phone interviews. Transcripts were analyzed using rapid content analysis with key practices and issues synthesized in profile summaries. Themes were identified and iteratively revised, utilizing matrices to compare content across early- and late-adopting sites.

KEY RESULTS: Five successful clinical practices were identified (use of two specific screening tools for primary IPV screening and secondary risk assessment, multilevel resource provision and community partnerships, colocation of mental health/social work, and patient-

Received September 17, 2018

Revised February 27, 2019

Accepted May 23, 2019

Published online August 16, 2019 centered documentation). Multilevel barriers (time/resource constraints, competing priorities and mounting responsibilities in primary care, lack of policy, inadequate training, and discomfort addressing IPV) and facilitators (engaged IPV champions, internal and external supports, positive feedback regarding IPV screening practices, and current, national attention to violence against women) were identified.

CONCLUSIONS: Findings advance national efforts by highlighting successful clinical practices for IPV screening programs and informing strategies useful for enhancing their implementation within and beyond the VHA, ultimately improving services and women's health.

KEY WORDS: evidence-based medicine; healthcare response; intervention; implementation science; qualitative research; women Veterans.

J Gen Intern Med 34(11):2435-42

DOI: $10.1007 / \mathrm{s} 11606-019-05240-\mathrm{y}$

(c) Society of General Internal Medicine (This is a U.S. government work and not under copyright protection in the U.S.; foreign copyright protection may apply) 2019

I ntimate partner violence (IPV) against women is a population health problem with wide-reaching impact. ${ }^{1-3}$ In the United States (US), one in three women experiences physical aggression, sexual violence, or stalking by an intimate partner. ${ }^{4}$ The numerous and often debilitating health outcomes from IPV include direct injury and poorer physical and psychological health and overall well-being. ${ }^{5-7}$

Women who experience IPV are frequently seen in primary care (PC), creating opportunity for identification and intervention. ${ }^{8,9}$ The US Preventative Services Task Force (USPSTF) and recent reviews found evidence that screening paired with 
response interventions can reduce IPV and attendant physical and mental health harms. ${ }^{10-12}$ Routine screening promotes readiness to disclose and increases access to IPV-related services. ${ }^{13}$ Additionally, women who discussed IPV with a provider were four times more likely to use an intervention and 2.6 times more likely to exit the relationship. ${ }^{14}$ Given the high prevalence of IPV, many women could benefit from effective implementation of healthcare-based IPV screening programs, but there is limited evidence on how to effectively implement screening recommendations in real-world care.

IPV is a critical issue for the Veterans Health Administration (VHA), as women Veterans are at higher risk for IPV than women who never served in the military. ${ }^{15}$ Findings from a national survey of VHA PC patients revealed that nearly one in five women experienced past-year IPV. ${ }^{16}$ Additionally, non-Veteran women who use VHA PC (i.e., spouses, dependents of service-connected Veterans) are at high risk for IPV. ${ }^{17}$ In response to these findings, and consistent with USPSTF recommendations, ${ }^{10}$ the VHA disseminated recommendations for IPV screening within PC in 2014. ${ }^{18}$ Even though the VHA recommends use of a validated IPV screening tool, ${ }^{19}$ and an on-site IPV Coordinator (IPVC) to assist with implementation and IPV-related care, the uptake of IPV screening program recommendations and best clinical practices throughout this large integrated healthcare system remains unknown.

As widespread IPV screening is in its infancy, there are lessons to learn from VA Medical Centers' (VAMC) screening adoption that can inform the scale-up of successful practices within the VHA, and beyond. In this study, we (a) characterized successful clinical practices of IPV screening programs in the VHA and (b) identified multilevel barriers to and facilitators of IPV screening programs.

\section{METHODS}

\section{Design and Participants}

All study procedures were approved by the VA Boston Institutional Review Board. In 2016, 140 VAMCs responded to a national program evaluation conducted by VHA's leadership regarding IPV-related programming implementation. Women's health PC staff completed follow-up program evaluation surveys regarding current adoption status that provided the sampling pool for this study. Early-adopting sites were defined as those that reported currently engaging in IPV screening as part of routine care (i.e., screening all female patients of child-bearing age or all female patients at least annually). Late-adopting sites were defined as those that reported not currently engaging in IPV screening as routine care (i.e., no screening or screening at provider's discretion). This categorization resulted in a sampling pool of 25 VAMCs provided by VHA leadership. From this roster, 15 VAMCs were purposively targeted for potential recruitment based on IPV screening adoption status and geographic location. ${ }^{20}$ We attempted to recruit 13 of these VAMCs, 2 of which declined to participate. We recruited sites until saturation was reached, totaling 11 VAMCs (6 early- and 5 late-adopting sites).

We recruited key informants for individual phone interviews at each site based on known VHA structures and roles involved in IPV screening programs. A total of 32 key informants were interviewed (74\% participation rate), including IPV Coordinators (IPVC; $n=9$ ), Women Veteran Program Managers (WVPM; $n=7$ ), Women's Health Medical Directors (WHMD; $n=6$ ), Psychologists $(n=4)$; Social Workers (SW; $n=3$ ), and Women's Health PCPs (WH PCP) or Nurse Care Managers $(n=3)$. Table 1 displays positions/roles of key informants.

\section{Approach}

A semi-structured interview guide and codebook were developed based on the integrated-Promoting Action on Research Implementation in Health Services (i-PARIHS) framework, which emphasizes innovation characteristics, individuals (i.e., recipients in line to adopt the innovation and patients who receive the innovation), and inner and outter contextual factors that inhibit or enable implementation. ${ }^{21}$ Sample questions included "What is the process of IPV screening and response in your clinic?" and "What have been challenges to integrating IPV screening?" The first author conducted audio recorded interviews, which were transcribed verbatim. Four team members then independently coded the same transcripts using the deductive codebook, convened to discuss and refine codebook definitions, and reached consensus across codes and transcripts through hybrid inductive-deductive rapid content analysis. ${ }^{22}$ Thereafter, transcripts were independently coded by two team members and coding was discussed until consensus was achieved. Using NVivo software, ${ }^{23}$ team members pulled coded content from all transcripts and created individ-

Table 1 Key Informants and Respective Roles $(N=32)$

\begin{tabular}{|c|c|c|}
\hline Position & Acronym & Role in VA \\
\hline Women's Health & WHMD & Internist, family practice \\
\hline Medical Director & & physician, or gynecologist \\
\hline $\begin{array}{l}\text { Women's Health } \\
\text { Primary Care }\end{array}$ & WH PCP & $\begin{array}{l}\text { General internist, family practice } \\
\text { physician or nurse practitioner/ }\end{array}$ \\
\hline Physician & & $\begin{array}{l}\text { physician assistant with } \\
\text { credentialing in women's health }\end{array}$ \\
\hline $\begin{array}{l}\text { Women's Health } \\
\text { Nurse Care Manager }\end{array}$ & N/A & $\begin{array}{l}\text { Registered nurse who assists in } \\
\text { managing/coordinating complex } \\
\text { care needs of women Veterans }\end{array}$ \\
\hline Psychologist & N/A & $\begin{array}{l}\text { Psychologists working in a } \\
\text { women's health clinic or directly } \\
\text { with a women's health team }\end{array}$ \\
\hline Social Worker & SW & $\begin{array}{l}\text { Attached to or working with a } \\
\text { women's health primary care team }\end{array}$ \\
\hline $\begin{array}{l}\text { Women Veterans } \\
\text { Program Manager }\end{array}$ & WVPM & $\begin{array}{l}\text { Oversee provision of care to } \\
\text { women Veterans across the } \\
\text { VAMC to ensures access and } \\
\text { quality (typically an RN, SW, or } \\
\text { psychologist) }\end{array}$ \\
\hline IPV Coordinator & IPVC & $\begin{array}{l}\text { Recommended position at all } \\
\text { VAMCs. Typically, a licensed } \\
\text { SW responsible for coordinating } \\
\text { IPV education and response } \\
\text { efforts across a VAMC }\end{array}$ \\
\hline
\end{tabular}

VAMC, VA Medical Center 
ual site profiles to synthesize findings into themes with exemplar quotes. From site profiles, we used matrix analysis ${ }^{24}$ to display themes across all early- and late-adopting sites.

\section{RESULTS}

We identified five successful clinical practices from early adopters, as late adopters were in varying preimplementation stages. Early-adopting sites reported screening for 1.5 to 3.5 years. We also identified five barriers to and four facilitating factors of implementation from all sites, including factors that were unique to early- and late-adopting sites. Table 2 displays summarized findings.

\section{Successful Clinical Practices}

Successful clinical practices were defined as feasible, effective procedures and processes that are considered acceptable to providers and patients.

The Extended-Hit, Insult, Threaten, Scream (E-HITS) tool (a 5-item IPV screening tool validated for use with female VHA patients) $)^{19,25}$ was routinely administered in all early-adopting sites as a primary means of IPV detection, usually as a clinical reminder.

The 3-item risk assessment tool (adapted from the lengthier Danger Assessment), ${ }^{26}, 27$ administered succeeding positive detection of IPV (IPV+) for further assessment of severe violence was viewed as helpful for assessing risk, triaging, and safety planning.

Multilevel resource provision and community partnerships were critical following IPV screening, entailing internal (e.g., referrals to support groups/programs and mental health) and external (e.g., National Domestic Violence Hotline, community organizations) assistance for women at risk for IPV. Sites

Table 2 Summary of Primary Findings

\begin{tabular}{|c|c|}
\hline $\begin{array}{l}\text { Successful Clinical } \\
\text { Practices }\end{array}$ & $\begin{array}{l}\text { - The Extended-Hit, Insult, Threaten, Scream } \\
\text { (E-HITS) tool } \\
\text { - A 3-item risk assessment following disclo- } \\
\text { sures } \\
\text { - Multilevel resource provision and community } \\
\text { partnerships } \\
\text { - Co-location of social work and/or mental } \\
\text { health services } \\
\text { - Patient-centered documentation }\end{array}$ \\
\hline Barriers & $\begin{array}{l}\text { - Time and resource constraints } \\
\text { - Competing priorities and mounting } \\
\text { responsibilities within primary care } \\
\text { - Lack of formal policy or mandate on IPV } \\
\text { screening and response procedures } \\
\text { - Lack of or inadequate training on successful } \\
\text { clinical practices for IPV screening and } \\
\text { response } \\
\text { - Discomfort around (and avoidance of) } \\
\text { discussing IPV }\end{array}$ \\
\hline Facilitating factors & $\begin{array}{l}\text { - Engaged and supportive IPV champions } \\
\text { - Internal and external supports } \\
\text { - Positive feedback regarding IPV screening } \\
\text { practices } \\
\text { - Recognition of violence against women as a } \\
\text { current, national topic }\end{array}$ \\
\hline
\end{tabular}

relied on community partnerships and programs as supplemental supports (e.g., in-depth safety planning, legal advice, shelters). An IPVC explained:

We have several resources in our office on where they can get help. I've never had anybody want to go to a shelter from here, although I am prepared for that. We discuss safety planning and resources. We have a card on where to get help, including shelters and the Domestic Violence Hotline.

Co-location of social work and/or mental health services enhanced IPV-related care. Participants underscored that Primary Care Mental Health Integration (PCHMI) staff are ideally co-located; their visibility, open access schedules, and expertise ensure timely warm handoffs and instill provider comfort and continuity of care. As expressed by an IPVC, "Because I'm co-located in primary care, it's such a faster track to therapy if somebody needs that. We don't necessarily have to refer out or have the Veteran repeat her story."

Patient-centered documentation of IPV-related care (e.g., positive screens, disclosures, and referrals), consisting of conversations with patients about what they are comfortable documenting in their electronic medical records, was a key theme. Such documentation was sensitive to patient safety and preferences. As explained by a SW, "Is it safe to document in the record?' is asked during screening. If it's not, we do not explicitly document IPV in the patient's electronic medical record." Nonetheless, sites acknowledged the need for continued education to sustain patient-centered documentation.

\section{Barriers}

Barriers were defined as short- and long-term challenges to implementation and/or maintenance of IPV screening programs.

Time and Resource Constraints. Time-related constraints cited among early- and late-adopting sites included limited time within an appointment to address both medical issues and an IPV+ screen. Resource constraints included insufficient availability of community (e.g., domestic violence agencies) and hospital (e.g., staffing shortages, limited availability of essential staff (e.g., IPVC) supports. A psychologist explained:

We've had chronic staffing shortages the last couple of years, coinciding with the IPV screening initiative coming from the national program ... One of the main concerns is still 'who will be responsible for following up on positive screens and where?' and then 
identifying what services to connect people to. There's infrastructure building and provider education that needs to happen, and there hasn't been a champion on site with time devoted to putting all those pieces together yet.Unique to late-adopting sites was the insufficient time dedicated to the IPVC role, which was a collateral duty to their existing job, with limited or no dedicated time. This severely limited availability for IPV screening program tasks.

\section{Competing Priorities and Mounting Responsibilities Within} Primary Care. PC was viewed as the "gatekeeper" for new initiatives, and their providers a "catch-all" for any residual needs of patients, especially within women's health. The numerous clinical reminders and high workload assigned to PC can result in "reminder fatigue" and burnout among providers, which can negatively impact IPV screening uptake and practices. An Associate Chief of SW stated:

[Providers] have all these clinical reminders they have to get done and they can't even get down to the reason for the appointment until these reminders are knocked out of the way, and the more clinical reminders you add to them, ... They become desensitized to the purpose behind it.Similarly, integrating IPV screening is sometimes viewed as an additional stressor for those who treat complex patient populations within busy PC settings. A WHMD highlighted the burden on providers:

You have all these screens that have been triggered positive where the provider needs to follow up, but the patient is there about their ankle ... Then your 8:30 has arrived, and your 8:00 is in the 8:30 time slot. You live under that tremendous pressure and then you have instant messaging and medical advice line calls and then someone's walked in pregnant. So I've been reluctant to tell my providers that [IPV screening] is coming.Unlike early adopters, a few late-adopting sites perceived additional burden placed on WH PCPs due to the physical and mental health complexities of the patient population. Being a WH PCP was regarded as more challenging (and with higher turnover rates), compared with non-WH PCPs.

Lack of Formal Policy or Mandate on IPV Screening and Response Procedures. Frustration over lags in "forthcoming" directives and national clinical reminders left some sites, especially late-adopting sites, stalled in negotiations with local leadership for resources to implement IPV screening programs. Some late-adopting sites explained that lack of leadership support surrounding the IPV initiative as an impediment for pushing IPV screening forward. Sites anticipate that having an official directive or mandate would provide clear expectations for developing protocols and delineating responsibilities. PC leadership at late-adopting sites was less receptive to the IPV screening initiative coming from SW in the absence of such formal policy. A WVPM stated:

VA needs to be more definitive about exactly what they want done and have a clear expectation for doing it. Often they'll put on initiatives, but there's no push for it, so two years later we're still trying to do it and it's okay because nobody ever gave us a hard date to get it done by.At the same time, a few sites posited that recommendations from agency leadership may underestimate day-to-day challenges at individual sites. A WHMD expressed concerns that site-specific time and resource realities may not support forthcoming directives: "I think that when these things come down from way up high I'm not certain that the reality of what happens in the clinic day-to-day is taken into consideration."

Lack of or Inadequate Training on Successful Clinical Practices for IPV Screening and Response. Sites discussed inadequate IPV training in providers' respective professional training programs, as well as ongoing education needed in their current roles to better screen and respond to IPV. A SW reflected:

I offered some on the side training with our gynecologists. One of the things that just floored me whenever I was doing this training is that there is no mention of assessment for IPV within the medical school for physicians who are going to be the people examining the general well-being of our patients. They've received no training. Sites also relayed instances of staff lacking knowledge of trauma-sensitive care, creating a potentially unsafe environment for implementing IPV screening programs. A WVPM discussed the implications of limited training on trauma-informed documentation:

Trainings cover how to protect the patient's privacy with documentation, because we're concerned about partners having access to online patient records. Before implementing this program, we had a doctor insistent on documenting what shelter this woman was at, and of course, the women's health SW hit the roof and educated them. 
Discomfort Around (and Avoidance of) Discussing IPV. Provider discomfort with IPV was a barrier across disciplines and leadership levels. Effective trainings and awareness-raising activities were helpful in overcoming this barrier at early-adopting sites. Discomfort and avoidance were perceived as more salient or harder to overcome at lateadopting sites, especially among some male decision-makers in PC. An IPVC noted:

People around the table were male leaders ... They were turned off by IPV screening because it made them evaluate their own behavior and they didn't like it ... They basically brushed [implementation] off.

\section{Facilitating Factors}

Facilitators were defined as attributes that helped get IPV screening programs implemented and/or maintained.

Engaged and Supportive IPV Champions. Sites recognized IPV champions' roles in driving IPV screening program implementation, and in sustaining broader IPV-related activities (e.g., obtaining additional funding for positions, creating IPV work groups, initiating support/ skills groups for IPV+ women). Champions were often IPVCs, WHMDs, WVPMs, or SWs within WHPC. IPVC champions played a more prominent role in implementation at early-adopting sites, corresponding with more protected time for the role at these sites. Champions frequently facilitate and accept warm handoffs, provide on-site consultation, and reinforce provider willingness to engage in IPV screening practices. They represent well-connected resources and liaisons with national leadership. A psychologist asserted the value of an IPV champion in implementation efforts:

Our IPVC is very knowledgeable, approachable, and friendly. She is able to put time and energy into this topic and when she's present, you see people asking questions and getting involved. She can make relationships with people. ... She is critical to the success of the program.

Internal and External Supports. Early-adopting sites noted several internal and external supports underlying IPV screening implementation success. Along with the presence of an IPV champion, early-adopting sites noted internal support from local leadership as a catalyst for IPV screening program implementation. Additionally, early-adopting sites regarded effective trainings (e.g., trauma-informed screening procedures, general education, and role-playing) as an ongoing facilitator of IPV screening implementation. External supports included engagement with national IPV and women's health leadership. Specifically, IPVCs and WVPMs consistently liaise with internal and external leadership and staff to communicate program updates, relay bidirectional feedback, and troubleshoot challenges. An IPVC shared: I think the biggest thing is the collaboration between the people at different sites and national-level people as well. The best thing about the VA is our interconnectedness and ability to share lessons learned and contribute back to one another ... That's such a motivator.

Positive Feedback Regarding IPV Screening Practices. Positive experiences with and feedback about IPV screening and response practices from patients and staff helped to bolster support for the program, especially at early-adopting sites. For example, patients and staff endorse routine IPV screening (often the EHITS) for its ability to facilitate discussion on IPV subtypes and promote conversations about past or current relationship abuse/patterns. A WH PCP described the value of screening: "People see they're getting some relief, they appreciate it, because sometimes they have shame attached to it, and I feel like this means a burden has been taken off them." A psychologist underscored the value of screening while demonstrating VHA support of this health issue: "What I like is the E-HITS raises those questions and opens up a conversation with the Veteran....and sends the message that we take it seriously and that we're here to provide support to the extent Veterans want or need."

Recognition of Violence Against Women as a Current, National Topic. Both early- and late-adopting sites reported local impact from the recent increase of national media coverage on sexual abuse and IPV against women. Sites reported that this surge in IPV representation on national platforms legitimizes and, in some cases, prioritizes IPV as a relevant health issue to target in female patients. As described by a psychologist:

Violence against women is a public health issue. The numbers are staggering and we're just not doing a lot to really address and take it seriously, and you see that in sexual harassment in our media right now ... Most women have known this is going on forever and it's just getting a moment in the spotlight ... There is an uphill battle to keep IPV relevant and real and at the top of people's lists. And I think maybe now is a moment, I mean riding the wave of the public discussion.Building on this momentum and keeping IPV initiatives in line with public discussions of violence against women 
were described as strategies to facilitate adoption of IPV screening programs.

\section{DISCUSSION}

Clinicians in PC are increasingly called on to screen for IPV, yet little is known about what clinical practices are successfully implemented in real-world care and what contextual factors impede or facilitate their uptake. This qualitative research identified five successful clinical practices and five barriers to and four facilitators of implementing IPV screening programs into WH PC within the VHA, the US' largest integrated healthcare system. This research is innovative because it is guided by an established implementation framework (i-PARIHS), ${ }^{21}$ enabling identification of clinical practices and contextual factors that inform evidence-based strategies for scale-up of IPV screening programs in and beyond the VHA.

Our findings regarding successful clinical practices and factors impacting IPV screening program implementation share similarities to previously reported elements of effective IPV screening programs, ${ }^{12}$ and reinforce VHA recommendations. ${ }^{18}$ Successful practices include adoption of two evidence-based screening tools (e.g., E-HITS and a 3-item risk assessment). ${ }^{25-28}$ These findings extend prior quantitative studies that have supported the effectiveness of the E-HITS for identifying IPV+ women and the utility of a subsequent 3-item risk assessment to facilitate triage and intervention. ${ }^{29-31}$ Consistent with i-PARIHS's focus on the role of innovation characteristics and evidence in uptake of new practices, these tools may be candidates for use given their demonstrated effectiveness, feasibility, and acceptability among our participants. Such tools reportedly attenuated provider discomfort and avoidance of discussing IPV, had perceived positive effects on clinical care, and were regarded as facilitators of implementation. Thus, the testimonials provided in this study may help promote uptake of IPV screening in other settings.

Key informants also illustrated the importance of multilevel resource provision and community partnerships, and colocated SW or mental health services as fundamental practices/processes for IPV screening and response protocols. These findings reinforce the importance of a systems approach to integrating IPV screening programs into healthcare settings. ${ }^{32-34}$ However, IPVCs reportedly had less (or no) protected time at late-adopting sites, limiting their capacity to cultivate resources, partnerships, and collaboration across settings. Dedicated time for IPVCs could enhance implementation of successful clinical practices and reduce barriers related to time constraints and mounting responsibilities within PC. Co-located SW and mental health enabled accessible consultation, effective trainings, and easy access for patients to intervention/referral sources. ${ }^{12}$ In fact, support from mental health, especially PCMHI, was a key factor that differentiated early- and late-adopting sites. Moving forward, PCMHI should be an integral part of IPV screening program implementation, as this care model can assist with multilevel resources and community referrals, while providing a pathway for patient-centered interventions for women who experience IPV. $^{35}$

Notably, our findings uncovered the feasibility of patientcentered documentation from provider and administrator perspectives, extending prior research on patient preferences. ${ }^{13}$ Patient-centered documentation includes engaging in transparent conversations with patients about their documentation preferences related to IPV such as obtaining patient consent to have IPV-specific details in their electronic medical record or limiting documentation altogether. ${ }^{13}$ Such practices lend themselves to enhancing care coordination while empowering patients who have reservations about disclosing and/or seeking help due to privacy and safety concerns to access care. ${ }^{36}$ Given the complexities of documentation and barriers related to time constraints, lack of training, and discomfort with IPV, there is a need for further research regarding patient-centered and trauma-informed documentation practices. The VHA is currently developing templates and guidance on IPV-related documentation, which may serve as models for other health settings.

"Reminder fatigue" may inhibit IPV screening, especially in the absence of formal policy from VHA leadership. As observed in this study, strategies to address fatigue may include relaying patients' and early-adopting sites' positive regard for IPV screening to clinicians and leadership. As demonstrated by findings on barriers, some sites may need to overcome male leadership discomfort with, or dismissal of, addressing IPV in a healthcare system that predominantly treats men. Increased awareness of, and education about, IPV, in tandem with public attention to violence against women, may reduce this issue. Finally, the lack of formal policy requiring IPV screening programs was a particularly salient barrier in late-adopting sites. In January 2019, after the completion of this study, the VHA issued a policy directive to this effect. ${ }^{37}$ The current findings are therefore extremely timely and can help more PC clinics adopt IPV screening programs throughout the VHA and other integrated healthcare systems.

A supportive and highly visible IPV champion can break down barriers and foster enabling factors. IPVCs with dedicated time for their role could serve as clinical champions as IPV screening programs are scaled up in the VHA. IPVCs serve as internal facilitators who assist with "boots on the ground" efforts while working with national programs for bidirectional feedback and up-to-date policy guidance. ${ }^{38}$ They liaise with local and national leadership to communicate sitespecific challenges to enhance feasibility or acceptability of national guidelines, overcoming a barrier identified in this study. Given the modifiable nature of several identified barriers and facilitators, implementation facilitation is a promising strategy to enhance uptake of successful clinical practices found in this study in VHA PC nationwide. Consistent with i-PARIHS, ${ }^{21}$ implementation facilitation is a multi-faceted 
process of enabling and supporting clinics and facilities, and has been successfully used in PC to overcome barriers and leverage facilitators to foster implementation of evidencebased practices. ${ }^{39,}, 40$ Thus, implementation facilitation could serve as a strategy to achieve successful implementation of IPV screening programs nationally within the VHA. Such efforts are needed to "ride the wave" of national attention to IPV and keep IPV initiatives in line with public discussions of violence against women.

Study limitations highlight areas for future research. This study did not systematically collect information on structural factors (e.g., staffing mix) that may impact uptake. This is an important next step for quantitative and mixed method studies. Patient perspectives were not examined, but VHA patient preferences and outcomes with IPV screening and responses practices have been studied previously. ${ }^{13,36,41}$ Nonetheless, patient feedback and quality improvement are important aspects of implementation success and require future examination. ${ }^{42}$ This study was limited to IPV screening programs for women who experience IPV. There is also a need to extend this line of research within the VHA to men who experience IPV and individuals who engage in IPV. ${ }^{43}, 44$

\section{Conclusion}

While IPV screening is recommended, many healthcare settings continue to struggle with implementation as society begins to more openly discuss abuse and violence against women. This study of IPV screening program implementation in the largest integrated healthcare system in the US provides important insights and clinical practices that can be applied broadly within and outside VHA settings, mitigating identified barriers.

Future directions include evaluations of implementation impact (e.g., reach, costs, sustainability) and clinical effectiveness (e.g., disclosure rates, post-screening IPV-related services use, satisfaction) of IPV screening programs in a wider array of PC clinics treating women and men. Combined with other IPV and implementation science literature, these findings provide actionable strategies that can facilitate effective IPV screening and intervention programs within PC settings. As US society grapples with the revelations of the \#MeToo movement, and greater disclosures of interpersonal violence and its attendant health effects, it is even more critical to effectively address IPV and to disseminate successful practices across health systems.

Acknowledgments: Our special thanks to Rachel M. Maskin for her assistance with data analysis and to the clinicians and administrators who shared their perspectives for this research. We also thank VHA's Office of Women's Health Services and the VHA IPV Assistance Program of Care Management and Social Work Services for providing program evaluation metrics for sampling and recruitment (Drs. Patricia Hayes, Sally Haskell, Galina Portnoy, and LeAnn Bruce).

Funding Support: This research was supported by the Department of Veterans Affairs, Veterans Health Administration, Health Services
Research and Development (HSR\&D) Services (PPO 17-044; PI Iverson). This work was also supported, in part, by Dr. Iverson's Presidential Early Career Award for Scientists and Engineers (USA 14-275) and Drs. Iverson's and Gutner's Implementation Research Institute fellowships from NIMH (5R25MH08091607) through HSR\&D Services, Buality Enhancement Research Initiative.

Corresponding Author: Katherine M. Iverson, $\mathrm{PhD}$; Women's Health Sciences Division, National Center for PTSDVA Boston Healthcare System, South Huntington Avenue, Boston, MA, USA (e-mail: katherine.iverson@va.gov).

\section{Compliance with Ethical Standards:}

Conflict of Interest: The authors declare that they do not have a conflict of interest.

Disclaimer: The views expressed in this article are those of the authors and do not necessarily reflect the position or policy of the Department of Veterans Affairs or the United States government.

\section{REFERENCES}

1. Bonomi AE, Thompson RS, Anderson M, et al. Intimate partner violence and women's physical, mental, and social functioning. American Journal of Preventive Medicine. 2006;30(6):458-466.

2. Devries KM, Mak JYT, Garcia-Moreno C, Petzold M, Child JC, Falder G, Lim S, Bacchus LJ, Engell RE, Rosenfeld L, Pallitto C, Vos T, Abrahams N, Watts CH. The Global Prevalence of Intimate Partner Violence Against Women. SCIENCE. 2013;340:1527-1528.

3. Liebschutz JM, Rothman, EF. Intimate-Partner Violence- What Physicians Can Do. New England Journal of Medicine. 2012;367(22).

4. Smith SG, Chen J, Basile KC, Gilbert LK, Merrick, MT, Patel N, Walling M, \& Jain A. National Intimate Partner and Sexual Violence Survey (NISVS) : 2010-2012 state report. 2017 (National Center for Injury Prevention and Control, Centers for Disease Control and Prevention, Atlanta, GA).

5. Campbell JC. Health consequences of intimate partner violence. The Lancet. 2002;359(9314):1331-1336.

6. Iverson KM, Dick A, McLaughlin KA, et al. Exposure to interpersonal violence and its associations with psychiatric morbidity in a U.S. national sample: A gender comparison. Psychology of Violence. 2013;3:273-287.

7. Hahn SA, Postmus JL. Economic empowerment of impoverished IPV survivors: a review of best practice literature and implications for policy. Trauma, Violence, \& Abuse. Apr 2014;15(2):79-93.

8. Ghandour RM, Campbell JC, Lloyd J. Screening and counseling for intimate partner violence: A vision for the future. Journal of Women's Health. 2015;24(1):57-61.

9. Kirst M, Zhang YJ, Young A, Marshall A, O'Campo P, Ahmad F. Referral to Health and Social Services for Intimate Partner Violence in Health Care Settings A Realist Scoping Review. Trauma, Violence, \& Abuse. 2012;13(4): 198-208.

10. Curry SJ, Krist AH, Owens DK, et al.,U.S. Preventive Services Task Force. Screening for Intimate Partner Violence, Elder Abuse, and Abuse of Vulnerable Adults: US Preventive Services Task Force Final Recommendation Statement. 2018.

11. Bair-Merritt MH, Lewis-O'Connor A, Goel S, et al. Primary care-based interventions for intimate partner violence: a systematic review. American Journal of Preventive Medicine. Feb 2014;46(2):188-194.

12. O'Campo P, Kirst M, Tsamis C, Chambers C, Ahmad F. Implementing successful intimate partner violence screening programs in health care settings: Evidence generated from a realist-informed systematic review. Social Science \& Medicine. 2011;72(6):855-866.

13. Iverson KM, Huang K, Wells SY, Wright JD, Gerber MR, WiltseyStirman S. Women veterans' preferences for intimate partner violence screening and response procedures within the Veterans Health Administration. Research in Nursing and Health. 2014;37:302-311.

14. McCloskey LA, Lichter E, Williams C, Gerber M, Wittenberg E, Ganz M. Assessing intimate partner violence in health care settings leads to women's receipt of interventions and improved health. Public Health Reports. 2006; 121(4):435-444.

15. Dichter ME, Cerulli C, Bossarte RM. Intimate Partner Violence Victimization Among Women Veterans and Associated Heart Health Risks. Women's Health Issues. 2011;21(4):S190-S194. 
16. Kimerling R, Iverson KM, Dichter ME, Rodriguez A, Wong A, Pavao J. Prevalence of Intimate Partner Violence among Women Veterans who Utilize Veterans Health Administration Primary Care. Journal of General Internal Medicine. 2016;31(8):888-894

17. Dichter ME, Haywood TN, Butler AE, Bellamy SL, Iverson KM Intimate partner violence screening in the Veterans Health Administration: Demographic and military service characteristics. American Journal of Preventative Medicine. 2017;52(6):761-768.

18. Veterans Health Administration Domestic Violence Task Force. Plan for Implementation of the Domestic Violence/Intimate Partner Violence Assistance Program. Department of Veterans Affairs, Veterans Health Administration;2013.

19. Iverson KM, King MW, Gerber MR, et al. Accuracy of an intimate partner violence screening tool for female VHA patients: A replication and extension. Journal of Traumatic Stress. 2015;28(1):79-82.

20. Palinkas LA, Horwitz SM, Green CA, Wisdom JP, Duan N, Hoagwood $\mathbf{K}$. Purposeful Sampling for Qualitative Data Collection and Analysis in Mixed Method Implementation Research. Administration and Policy in Mental Health and Mental Health Services Research. 2013;42(5):533-544.

21. Harvey G, Kitson A. PARIHS revisited: from heuristic to integrated framework for the successful implementation of knowledge into practice. Implementation Science. 2016;11(1): 1 .

22. Hamilton A. Qualitative methods in rapid turn-around health services research. VA HSR\&D. 2013.

23. NVivo qualitative data analysis Software; QSR International Pty Ltd Version 10, 2012.

24. Averill JB. Matrix analysis as a complementary analytic strategy in qualitative inquiry. Qualitative Health Research. 2002;12(6):855-866.

25. Chan C, Chan Y, Au A, Cheung G. Reliability and validity of the "Extended - Hurt, Insult, Threaten, Scream" (E-HITS) screening tool in detecting intimate partner violence in hospital emergency departments in Hong Kong. Hong Kong Journal of Emergency Medicine. 2010;17:109117.

26. Campbell JC. Danger Assessment-5. 2015.

27. Campbell JC, Webster DW, Glass N. The danger assessment: Validation of a lethality risk assessment instrument for intimate partner femicide. Journal of Interpersonal Violence. 2009;24(4):653-674.

28. Sherin KM, Sinacore JM, Li XQ, Zitter RE, Shakil A. HITS: A short domestic violence screening tool for use in a family practice setting. Journal of Family Medicine. 1998;30:508-512.

29. Dichter ME, Butler A, Bellamy S, Medvedeva E, Roberts CB, Iverson KM. Disproportionate mental health burden associated with past-year intimate partner violence among women receiving care in the Veterans Health Administration. Journal of Traumatic Stress. 2017;6:555-563.

30. Iverson KM, Sorrentino AE, Bellamy SL, et al. Adoption, penetration, and effectiveness of a secondary risk screener for intimate partner violence: Evidence to inform screening practices in integrated care settings. General Hospital Psychiatry. 2018;51:79-84.

31. Iverson KM, King M, Resick PA, Gerber MR, Kimerling R, Vogt D. Clinical utility of an intimate partner violence screening tool for female VHA patients. Journal of General Internal Medicine. 2013;28:1288-1293.

32. Miller E, McCaw B, Humphreys BL, Mitchell C. Integrating intimate partner violence assessment and intervention into healthcare in the
United States: A systems approach. Journal of Women's Health. 2015;24(1):92-99.

33. Decker MR, Frattaroli S, McCaw B, et al. Transforming the healthcare response to intimate partner violence and taking best practices to scale. Journal of Women's Health. 2012;21(12):1222-1229.

34. McCaw B. Using a Systems Model Approach to Improving IPV Services in a Large Health Care Organization. The National Academies Press. 2011.

35. Iverson KM, Wiltsey-Stirman $\mathbf{S}$, Street $\mathbf{A E}$, et al. Female veterans' preferences for counseling related to intimate partner violence: Informing patient-centered interventions. General Hospital Psychiatry. 2016;40:3338.

36. Dichter ME, Wagner C, Goldberg E, Iverson KM. Intimate Partner Violence Detection and Care in the Veterans Health Administration: Patient and Provider Perspectives. Women's Health Issues. 2015;25(5):555-560

37. Department of Veterans Affairs. Intimate Partner Violence Assistance Program, 10P4C - Care Management and Social Work Service. Directive 1198. 2019.

38. Ritchie MJ, Dollar KM, Miller $\mathbf{C J}$, et al. Using implementation facilitation to improve care in the Veterans Health Administration (Version 2). 2017; https://www.queri.research.va.gov/tools/implementation/Facilitation-Manual.pdf. Accessed 30 July 2018

39. Baskerville NB, Liddy C, Hogg W. Systematic Review and Meta-Analysis of Practice Facilitation Within Primary Care Settings. The Annals of Family Medicine. 2012;10(1):63-74

40. Kirchner JE, Ritchie MJ, Pitcock JA, Parker LE, Curran GM, Fortney JC. Outcomes of a partnered facilitation strategy to implement primary care-mental health. Journal of General Internal Medicine. 2014;29(Suppl 4):904-912.

41. Dichter ME, Sorrentino AE, Haywood TN, et al. Women's Healthcare Utilization Following Routine Screening for Past-Year Intimate Partner Violence in the Veterans Health Administration. Journal of General Internal Medicine. 2018;33(6):936-941.

42. Proctor E, Silmere H, Raghavan R, et al. Outcomes for implementation research: Conceptual distinctions, measurement challenges, and research agenda. Administration and Policy in Mental Health and Mental Health Services Research. 2011;38(2):65-76.

43. Iverson KM, Vogt D, Maskin R., \& Smith BR. Intimate partner violence victimization and associated implications for health and functioning among male and female post-9/11 veterans. Medical Care. 2017;55(9):S78-S84.

44. Portnoy GA, Haskell SG, King MW, Masking R, Gerber MR, \& Iverson KM. Accuracy and acceptability of a screening tool for identifying intimate partner violence perpetration among women veterans: A preimplementation evaluation. Women's Health Issues. 2018;28(5):439-445.

Publisher's Note Springer Nature remains neutral with regard to jurisdictional claims in published maps and institutional affiliations. 